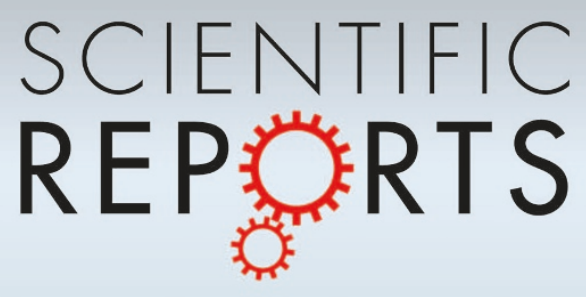

OPEN

SUBJECT AREAS:

PHASE TRANSITIONS

AND CRITICAL

PHENOMENA

ELECTRONIC PROPERTIES AND MATERIALS

Received

2 August 2013

Accepted

6 September 2013

Published

2 October 2013

Correspondence and requests for materials should be addressed to

R.Y. (ryoshida@issp.u-

tokyo.ac.jp) or T.Y. (yokoya@cc.okayamau.ac.jp)

\section{Translational Symmetry Breaking and Gapping of Heavy-Quasiparticle Pocket in $\mathrm{URu}_{2} \mathrm{Si}_{2}$}

Rikiya Yoshida', Koji Tsubota', Toshihiko Ishiga' ', Masanori Sunagawa', Jyunki Sonoyama', Dai Aoki², Jacques Flouquet ${ }^{3}$, Takanori Wakita' ${ }^{1}$ Yuji Muraoka' \& Takayoshi Yokoya'

'Graduate School of Natural Science and Technology, Okayama University, Okayama 700-8530, Japan, ${ }^{2}$ INAC/SPSMS, CEA-Grenoble, Grenoble 38054, France and IMR, Tohoku University, Oarai, Ibaraki 31 1-1313, Japan, ${ }^{3}$ INAC/SPSMS, CEAGrenoble, Grenoble 38054, France.

$\mathrm{URu}_{2} \mathrm{Si}_{2}$ is a uranium compound that exhibits a so-called 'hidden-order' transition at $\sim 17.5 \mathrm{~K}$. However, the order parameter of this second-order transition as well as many of its microscopic properties remain unclarified despite considerable research. One of the key questions in this regard concerns the type of spontaneous symmetry breaking occurring at the transition; although rotational symmetry breaking has been detected, it is not clear whether another type of symmetry breaking also occurs. Another key question concerns the property of Fermi-surface gapping in the momentum space. Here we address these key questions by a momentum-dependent observation of electronic states at the transition employing ultrahigh-resolution three-dimensional angle-resolved photoemission spectroscopy. Our results provide compelling evidence of the spontaneous breaking of the lattice's translational symmetry and particle-hole asymmetric gapping of a heavy quasiparticle pocket at the transition.

$\mathrm{n}$ the field of strongly correlated electron systems, the term 'hidden order' is often used to refer to a secondorder phase transition whose order parameter has not yet been identified. One of the most well-known examples of this phenomenon is the 'hidden-order' transition in $\mathrm{URu}_{2} \mathrm{Si}_{2}$. This transition shows clear anomalies in terms of specific heat and transport data at $T_{\mathrm{HO}}=\sim 17.5 \mathrm{~K}^{1-3}$. However, previous studies have found no experimental indication of structural transition or conventional intrinsic magnetic order. Although a neutron scattering experiment detected a small ordered moment $\left(\sim 0.03 \mu_{B}\right.$ per uranium atom, where $\mu_{B}$ represents the Bohr magneton) below $T_{\mathrm{HO}}{ }^{4}$, the magnitude was too small to explain the large entropy release upon the transition. This small moment is now ascribed to an extrinsic effect ${ }^{5}$. Many theoretical models have been proposed to explain this mysterious order ${ }^{6-16}$; nonetheless, various important questions remain to be addressed experimentally.

The key questions with regard to this mysterious second-order transition are the type of spontaneous symmetry breaking and the property of Fermi-surface gapping in the momentum space. A recent magnetic torque experiment reported the breaking of four-fold rotational symmetry upon the transition ${ }^{17}$; however, the existence of other types of symmetry breaking remains an open question. In particular, while scanning tunneling spectroscopy (STS) studies ruled out electronic periodicity modulation within the $a-b$ plane $\mathrm{e}^{18,19}$, possible translational symmetry breaking along the $c$ axis (or $k_{\mathrm{z}}$ direction in the momentum space) has been indirectly suggested by neutron scattering ${ }^{20}$ and further supported by quantum oscillation ${ }^{21}$. (Figure S1(a) in the 'Supplementary Information' illustrates the crystal structure of $\mathrm{URu}_{2} \mathrm{Si}_{2}$.) Despite no experimental indication of structural change, a spectroscopic study may still be beneficial because the change in electronic states can be more drastic. As for the Fermi-surface gapping, two complementary spectroscopic probes have not revealed any consistent information: STS showed a gap opening at the Fermi level in the local density of states ${ }^{18,19}$, but angle-resolved photoemission spectroscopy (ARPES), a momentum-space counterpart of STS, did not ${ }^{22-25}$. Therefore, although momentumdependent electronic states have been reported, more detailed investigations both in the paramagnetic state and in the hidden-order state are necessary to address the above-mentioned key questions.

To study the momentum-dependent modifications of electronic states as a function of temperature, we employed ultrahigh-resolution three-dimensional ARPES. The tuning of excitation-photon energy allows us to probe various $k_{\mathrm{z}}$ values in the Brillouin zone of $\mathrm{URu}_{2} \mathrm{Si}_{2}$. (The Brillouin zone is illustrated in Fig. S1(b) in the 'Supplementary Information'.) Moreover, previous studies that reported the observation of a narrow band ${ }^{22,23}$ have indicated that meV-scale changes near the Fermi level can be important. Therefore, for our research 
purposes, we performed our experiments on high-quality single crystals at an ultrahigh-resolution ARPES endstation located at a thirdgeneration synchrotron source.

\section{Results}

Paramagnetic state. Photon-energy-dependent measurements in the paramagnetic state reveal several important insights into the observed band structures. Figure 1 shows ultrahigh-resolution ARPES data for the $\Gamma$-X direction recorded at $20 \mathrm{~K}$. We used $h v \sim 19 \mathrm{eV}$ and $\sim 34 \mathrm{eV}$ to probe the $\Gamma$ point and the $\mathrm{Z}$ point, respectively ${ }^{26}$. The upper panel shows data measured using $19-\mathrm{eV}$ photons; the intensity map in Fig. 1(a) shows a hole pocket $\left(k_{\mathrm{F}} \sim 0.16 \AA^{-1}\right)$ as well as a holelike dispersive band whose top is well below the Fermi level. In addition, the corresponding energy-distribution curves (EDCs) in Fig. 1(b) and the momentum-distribution curves (MDCs) in Fig. 1(c) consistently provide evidence for these bands. The lower panel shows ARPES data measured using 34-eV photons; the intensity map in Fig. 1(d) clearly shows a hole-like band whose top exists well below the Fermi level, and the MDCs in Fig. 1(f) show the existence of a hole pocket. While the hole-like band observed at two different photon energies was experimentally shown as a surfaceoriginated state ${ }^{22,27}$, the hole pocket is also suggested as a surfacederived state owing to the absence of $k_{\mathrm{z}}$ dependence and its inconsistency with bulk-sensitive measurements (see Supplementary Fig. S2 and Supplementary Discussion 1 for details). Therefore, unlike previous studies that regarded the hole pocket as a bulk state, we suggest that the hole pocket is not related to the hidden-order transition.

One striking observation in our data is the existence of an additional structure in the $34-\mathrm{eV}$ data. Although this additional structure was reported as a broad feature near the Fermi level ${ }^{24,25}$, the EDCs in Fig. 1(e) clearly show for the first time that the peak disperses toward the Fermi level as $\left|k_{110}\right|$ increases; this observation directly evidences the existence of an electron-like band. Furthermore, the MDCs in Fig. 1(f) suggests that this electron-like band crosses the Fermi level at the wave vector $k_{\mathrm{F}} \sim 0.30 \AA^{-1}$. To further confirm this picture, ARPES data normalized by resolution-convoluted Fermi functions are shown in Supplementary Fig. S3; these data indicate that (1) no flat feature is discernible both above and below the Fermi level in the $19-\mathrm{eV}$ data and (2) the electron band observed in the 34-eV data crosses the Fermi level at a wave vector consistent with the MDCderived $k_{\mathrm{F}}$ value (see Supplementary Discussion 2 for additional notes). In addition, because the electron band is $k_{\mathrm{z}}$ dependent, it should be assigned as a bulk state. We note that the observation of a $k_{\mathrm{z}}$-dependent, well-defined dispersion of electron pocket is a new experimental insight which was not known previously.

Hidden-order state. Entering the hidden-order state results in an abrupt change in the electronic structures near the Fermi level. Figure 2(a) and 2(d) show the ARPES intensity maps measured at $2 \mathrm{~K}$ by 19 - and $34-\mathrm{eV}$ photons, respectively; both maps show a narrow 'M-shaped' band near the Fermi level, a hole-like band, and a hole pocket, of which the last two are irrelevant to the

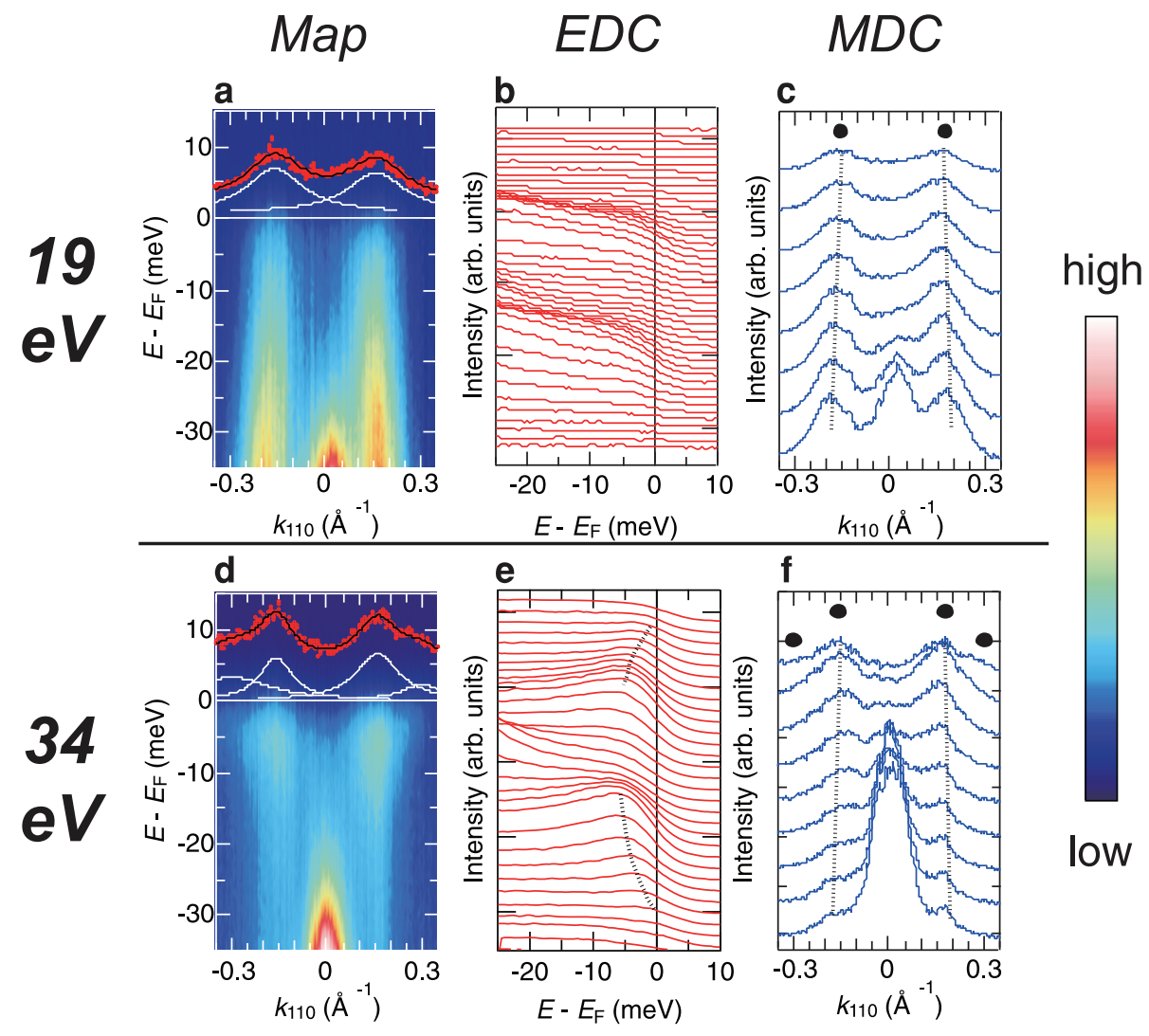

Paramagnetic State

Figure 1 High-resolution ARPES data measured along the $\Gamma$-X direction for the paramagnetic state of $\mathrm{URu}_{2} \mathrm{Si}_{2}$ (20 K). (a) ARPES intensity map measured using $19-\mathrm{eV}$ photons. The momentum-distribution curve (MDC) at the Fermi level fitted with Lorentzian functions is also shown.

(b) The energy distribution curves (EDCs) corresponding to the data in (a). (c) The MDCs corresponding to (a). (d) ARPES intensity map measured using 34-eV photons. The MDC at the Fermi level fitted with Lorentzian curves is also shown. (e) EDCs corresponding to (d). (f) MDCs corresponding to (d). Note that the positions of $k_{\mathrm{F}}$ are indicated by dots in (c) and (f), and the broken lines in (c), (e) and (f) are guides for the eye. 


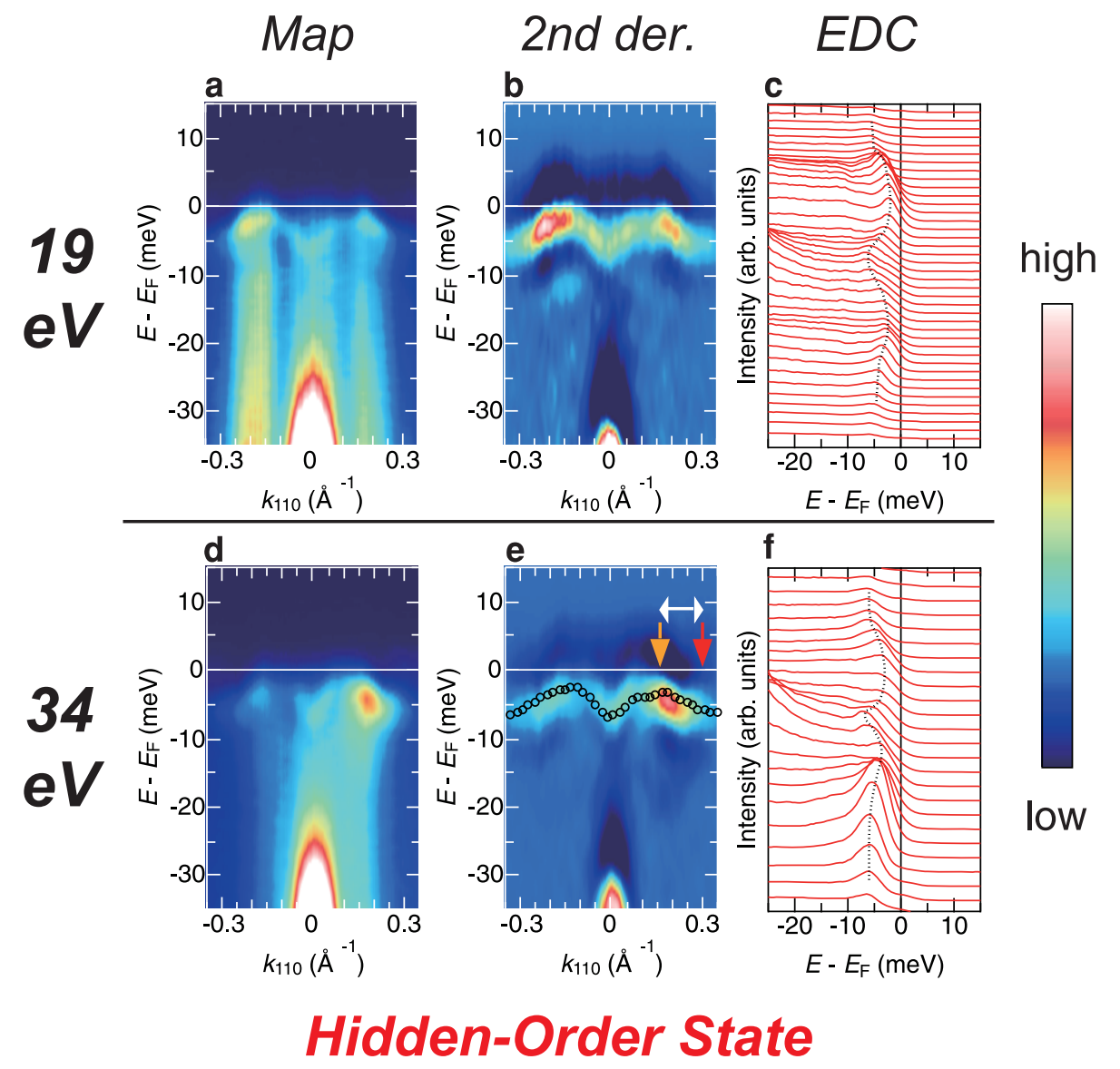

Figure $2 \mid$ High-resolution ARPES data measured along the $\Gamma$-X direction for the hidden-order state of $\mathrm{URu}_{2} \mathrm{Si}_{2}$ (2 K). (a) ARPES intensity map measured using 19-eV photons. (b) The second-derivative map corresponding to (a). (c) The energy-distribution curves corresponding to (a). (d) ARPES intensity map measured using $34-\mathrm{eV}$ photons. (e) The second-derivative map corresponding to (d). Black circles show the EDC-derived peak positions of the 'M-shaped' band in the $19-\mathrm{eV}$ data. The red arrow indicates the position of $k_{\mathrm{F}}$, and the orange arrow shows the top of the 'M-shaped' band. (f) The energy-distribution curves corresponding to (d). The broken lines in (c) and (f) are guides for the eye. Note that the spectra were measured with circular polarization.

hidden-order transition. In the corresponding second-derivative maps shown in Fig. 2(b) and 2(e), the 'M-shape' of the band is more clearly resolved. Moreover, the EDCs in Fig. 2(c) and 2(f) do not support the Fermi-level crossing of the 'M-shaped' band because well-defined peaks appear below the Fermi level. This narrow ' $\mathrm{M}$ shaped' band is similar to the one observed in the previous highresolution studies ${ }^{22-25}$; however, it should be pointed out that while no flat dispersive feature was observed in the $19-\mathrm{eV}$ data at $20 \mathrm{~K}$, the 'M-shaped' band suddenly becomes observable by the use of $19-\mathrm{eV}$ photons in the hidden-order state. In addition, we note that the 'Mshaped' band has its top at $k_{\text {top }} \sim 0.16 \AA^{-1}$ (indicated by the orange arrow in Fig. 2(e)) and disperses away from the Fermi level around $k_{\mathrm{F}}$ $\sim 0.30 \AA^{-1}$ (Fermi vector of the electron pocket indicated by the red arrow in Fig. 2(e)); this observation strongly suggests gap opening in the electron-like Fermi surface. Furthermore, because EDC-derived peak positions in the 19-eV data (black circles in Fig. 2(e)) match the dispersion in the $34-\mathrm{eV}$ data, it is suggested that these 'M-shaped' bands have the same origin.

Temperature-dependent electronic states. The temperature dependence of the photoemission spectra is shown in detail in Fig. 3, with an emphasis on how the 'M-shaped' band is formed through the transition. Figure 3(a) shows the temperature dependence of the second-derivative maps as measured using $34-\mathrm{eV}$ photons (the second-derivative maps are compared with the corresponding raw data in Supplementary Figure S4). Above the critical temperature, the renormalized electron pocket is observed below the Fermi level. The electron pocket starts to change its shape when cooled below the critical temperature, and further cooling results in the transformation of the electron pocket into an 'M-shaped' band. This temperature dependence confirms that the electron pocket is the origin of the ' $\mathrm{M}$-shaped' band; since the hidden-order transition involves $U 5 f$ electrons $^{2}$, it is suggested that the electron pocket originates from a $U 5 f$ state. In contrast, the temperature dependence of the intensity map as measured using $19-\mathrm{eV}$ photons in Fig. 3(b) shows that the intensity of the 'M-shaped' band appears and is enhanced only below the critical temperature.

The temperature dependence of EDCs, as shown in Fig. 3(c)-(f), further clarify the momentum-dependent changes of electronic states. The spectral cut at $k_{110}=0 \AA^{-1}$ as measured using $34-\mathrm{eV}$ photons, shown in Fig. 3(c), indicates a peak develops upon cooling at $E_{\mathrm{B}} \sim 7 \mathrm{meV}$. Moreover, at the Fermi wave vector of $k_{\mathrm{F}} \sim 0.30 \AA^{-1}$ (Fig. 3(d)), the leading edge shifts toward the higher $E_{\mathrm{B}}$, and a sharp peak develops at $E-E_{\mathrm{F}} \sim 7 \mathrm{meV}$; these observations further support the gapping of the electron pocket upon the hidden-order transition. In contrast to the 34-eV data, the EDCs of 19-eV data in Fig. 3(e) and Fig. 3(f) show that a peak appears both at $k_{110}=0 \AA^{-1}$ and at $k_{110}=$ $0.16 \AA^{-1}$ below the critical temperature. Moreover, the both peaks shift toward higher binding energies; although the shift is not clear for the cut at $k_{110}=0.16 \AA^{-1}$ owing to the thermal effect, the EDCs 

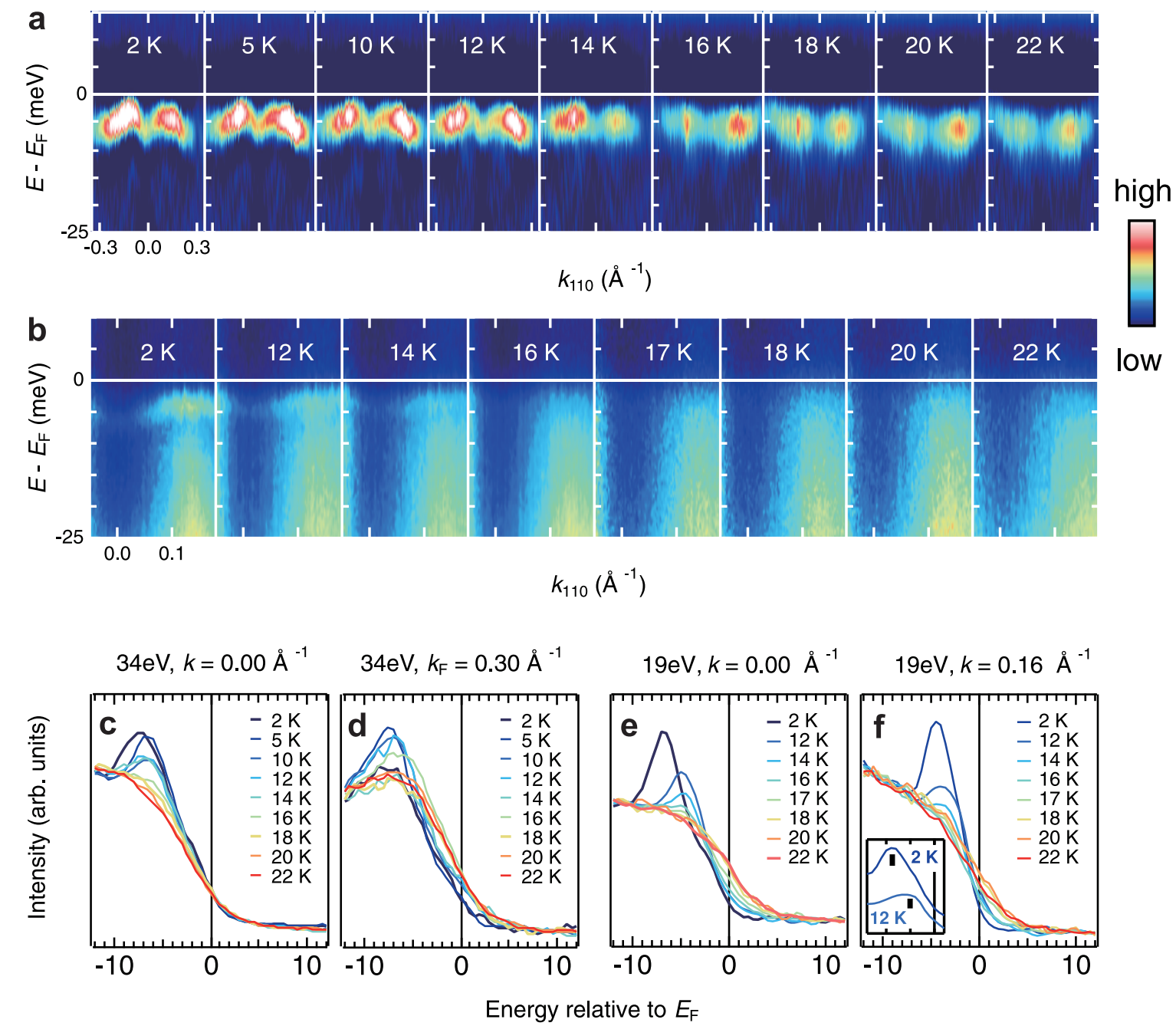

Figure $3 \mid$ Temperature dependence of 'M-shaped' band probed at two high-symmetry points. (a) Temperature dependence of the second-derivative maps measured using 34-eV photons (b) Temperature dependence of ARPES intensity maps measured using 19-eV photons. (c) The spectral cuts at $k_{110}=0 \AA^{-1}$ recorded using $34-\mathrm{eV}$ photons. (c) The spectral cuts at $k_{\mathrm{F}}=0.30 \AA^{-1}$ for $34 \mathrm{eV}$. (d) The energy-distribution curves at $k_{110}=0 \AA^{-1}$ for $19 \mathrm{eV}$. (f) The spectral cuts at $k_{110}=0.16 \AA^{-1}$ for $19 \mathrm{eV}$. The EDCs at $2 \mathrm{~K}$ and $12 \mathrm{~K}$ are also presented in the inset after being normalized by the resolutionconvoluted Fermi functions.

normalized by the resolution-convoluted Fermi fuctions clearly demonstrate the shift of the peak with temperature (see the inset of Fig. 3(f)). We note that this shift of the 'M-shaped' band is significantly different from the change of EDCs in the $34-\mathrm{eV}$ data. Therefore, the data strongly suggest that the evolution of electronic states at the hidden-order transition is $k_{\mathrm{z}}$ dependent.

\section{Discussion}

The major findings in the present study are summarized in Figure 4(a) and 4(b), from which we can learn about the nature of the hidden-order transition. In the paramagnetic state illustrated in Fig. 4(a), a renormalized electron-like Fermi surface exists around the $\mathrm{Z}$ point in the body-centered tetragonal Brillouin zone. We note that because no electron pocket is predicted at the $\mathrm{Z}$ point by localdensity approximation (LDA), LDA may be insufficient to describe some of the electronic states near the Fermi level. The electron-like Fermi surface is gapped out upon the transition, and it evolves into a narrow 'M-shaped' band, as illustrated in Fig. 4(b). This observation can be attributed to the observed decrease in the number of carriers ${ }^{28}$ and the development of a gap ${ }^{29}$. The observed gap is very peculiar because the top of the band after gapping $\left(k_{\text {top }} \sim 0.16 \AA^{-1}\right)$ is not aligned with the original Fermi vector $\left(k_{110} \sim 0.30 \AA^{-1}\right)$; this misalignment behaviour suggests that the gap is particlehole asymmetric. We note that similar misalignment is observed by ARPES for the case of a pseudogap in high- $T_{c}$ cuprates, and it evidences the opening of a particle-hole asymmetric gap ${ }^{30}$. The energy scale of the gap in $\mathrm{URu}_{2} \mathrm{Si}_{2}$ is of the order of $\sim 2-7 \mathrm{meV}$ (here, the lower limit is obtained from the leading-edge shift, and the upper limit from the peak position), which is in accordance with optical spectroscopy and specific heat ${ }^{3,29,31}$. Moreover, the in-plane wave-vector position of the observed gap $\left(k_{\mathrm{F}} \sim 0.30 \AA^{-1}\right)$ is in agreement with ultrafast time- and angle-resolved photoemission spectroscopy ${ }^{32}$.

The particle-asymmetric gap in the heavy electron pocket may be explained by the development of hybridization with a conduction band at $T_{\mathrm{HO}}$; however, we have the following remarks: (1) Our experimental data do not support the relevance of the hole pocket at $k_{110} \sim 0.16 \AA^{-1}$ to the bulk hybridization process at the transition. First of all, the hole pocket is suggested to be a surface-derived state, thus it should be irrelevant to the transition. In addition, if the light hole pocket at $k_{110}=0.16 \AA^{-1}$ hybridizes with the heavy electron pocket and forms a gap below the Fermi level, two split peaks would show up in the EDCs of $19-\mathrm{eV}$ data at $k_{110}=0.16 \AA^{-1}$ (Fig. 3(f)); however, we did not find any evidence for such split features below the Fermi level. Moreover, the wave vector of the hole pocket is 


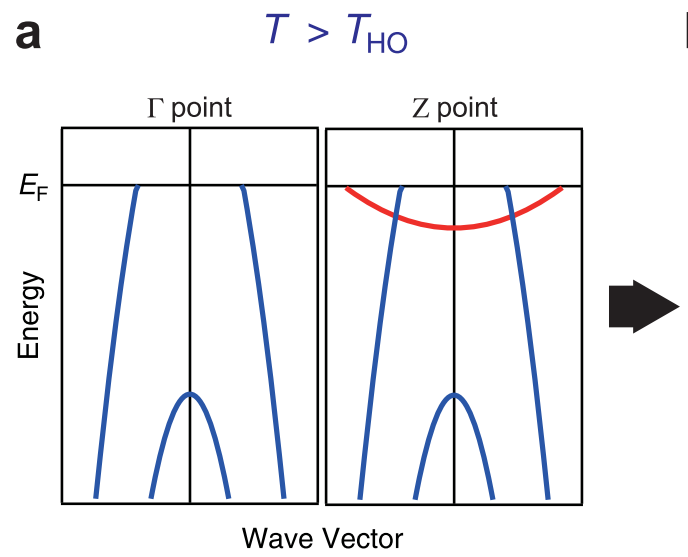

b $\quad T<T_{\text {HO }}$

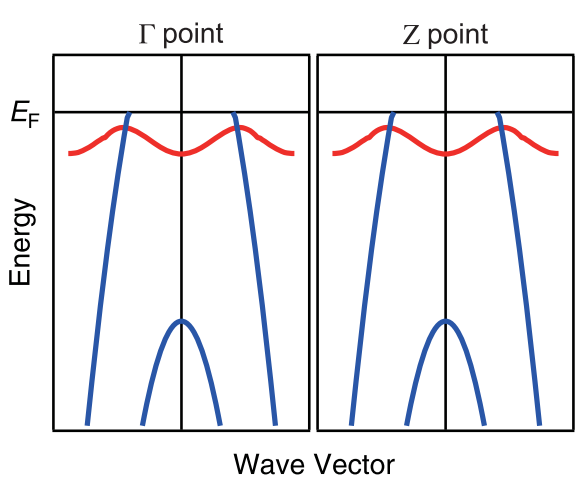

Figure 4 Schematic description of electronic states observed (a) in the paramagnetic phase and (b) in the hidden-order phase. Red lines represent bulk-originated states, and blue lines indicate $k_{z}$-independent states that are irrelevant to the hidden-order transition. The electron pocket at the $\mathrm{Z}$ point becomes gapped upon the transition and evolves into an ' $\mathrm{M}$-shaped' band. A similar narrow band appears at the $\Gamma$ point only in the hidden-order state, indicating the emergence of spontaneous translational symmetry breaking.

smaller than the value deduced in previous spectroscopic studies $^{18,19,33}$, further supporting the irrelevance. (2) Our data support that the energy gap is formed at the Fermi level upon the transition, and the 'M-shaped' band does not cross the Fermi level below $T_{\mathrm{HO}}$. This picture is consistent with the previous STS studies ${ }^{18,19}$, but is in contrast to the opening of a gap in momentum suggested by a recent ARPES study ${ }^{25}$. (3) Recall that a well-defined heavy fermion state which already exists above $T_{\mathrm{HO}}$ evolves into the 'M-shaped' band below $T_{\mathrm{HO}}$ around the $\mathrm{Z}$ point while no coherent state is observed around the $\Gamma$ point above $T_{\mathrm{HO}}$; moreover, our data indicate that the temperature evolution of ARPES spectra is significantly $k_{z}$ dependent. (Note that the $k_{\mathrm{z}}$-dependent temperature evolution cannot be explained by the mere intensity modulation due to the photoelectron matrix-element effect.) These observations cannot be explained only by the onset of hybridization between an incoherent (not welldefined) heavy fermion state and a conduction band at the transition temperature ${ }^{24}$. Therefore, although we do not exclude the role of 'spontaneous hybridization', we emphasize that the hybridization process between an incoherent state and a conduction band should not be the only effect at the transition. (4) There is no conclusive evidence for any bulk hole-like band being involved in the hybridization. One possible reconciliation is that another hole pocket remains undetected in ultrahigh-resolution ARPES owing to the matrix-element effect; another interesting possibility is that the ' $\mathrm{M}$ shaped' band is formed by a mechanism unique to a two-channel Kondo lattice ${ }^{33}$; this model does not necessarily require the existence of a hole pocket in the disordered state.

The 19-eV data show that the 'M-shaped' band appears only in the ordered state around the $\Gamma$ point. Although we observed the shift of 'M-shaped' band in the 19-eV data, its shape matches the dispersion observed in the 34-eV data when the order is well developed (say, at $\sim 2 \mathrm{~K}$ ); this observation strongly suggests that two high-symmetry points ( $\Gamma$ point and $\mathrm{Z}$ point) become equivalent in the ordered state, and the lattice's translational symmetry should be spontaneously broken along the $c$-axis with the ordering vector of $(0,0,1)$. This picture is further supported by the existence of a well-defined heavy fermion state only around the $\mathrm{Z}$ point in the paramagnetic state; the electronic states at the $\Gamma$ and the $\mathrm{Z}$ points are qualitatively different above $T_{\mathrm{HO}}$. The breaking of the translational symmetry along the $c$ axis is consistent with recent neutron data ${ }^{20}$, the reentrant behaviour of the hidden order ${ }^{34}$, and the similarity of the Fermi surfaces between the hidden-order state and the antiferromagnetic state ${ }^{21}$. Furthermore, as the present data show only electrons near the Fermi level change upon the transition, they may explain why $\mathrm{x}$ ray scattering could not detect translational symmetry breaking.
The present experimental results would impose strong constraints on the models of the hidden order. Our results may be attributable to an antiferro-type multipole ordering along the $c$-axis ${ }^{6-10,16}$; they are also in accordance with exotic Fermi-surface nesting instabilities, in which the nesting vector of $(0,0,1)$ plays a role $e^{11,12,15}$. However, we also note that an explanation beyond simple textbook-type band folding is necessary, as also pointed out recently ${ }^{22,25}$; future theoretical studies should address why the energy shift of the 'M-shaped' band around the $\Gamma$ point was observed. We expect that this study will promote a better understanding of complicated electronic states in $\mathrm{URu}_{2} \mathrm{Si}_{2}$ and also provide a framework for future studies on exotic phase-transition phenomena in correlated materials.

\section{Methods}

Preparation of $\mathrm{URu}_{2} \mathrm{Si}_{2}$ samples. The stoichiometric proportions of the starting ingredients (depleted uranium: 99.9\%, ruthenium: $99.99 \%$, and silicon: $99.999 \%$ ) were melted in a tetra-arc furnace, and single crystals of $\mathrm{URu}_{2} \mathrm{Si}_{2}$ were synthesized by the Czochralski method. The single-crystal ingot was cut using a spark cutter. The samples were annealed at $1075^{\circ} \mathrm{C}$ for 5 days under ultrahigh vacuum. The details are described in Ref. 35 . The high quality of the samples can be evidenced by quantum oscillation measurements ${ }^{21}$.

Ultrahigh-resolution three-dimensional ARPES. Ultrahigh-resolution threedimensional ARPES was performed at a 'One-Cubed' ARPES endstation located at BESSY II (Berliner Elektronenspeicherring Gesellschaft fur Syn-chrotronstrahlung). The spectrometer consists of a Gammadata VG-Scienta R4000 electron analyser and a continuous flow He-3 cryomanipulator. Photons from the UE112_PGM-2b beamline were used, and the total energy resolution was $4-7 \mathrm{meV}$ for $19-51 \mathrm{eV}$. The polarization of the incident photon was set to linear vertical unless otherwise noted. The analyser's slit was aligned along the [110] direction, which corresponds to the $\Gamma$ $\mathrm{X}$ direction in the body-centered tetragonal Brillouin zone of $\mathrm{URu}_{2} \mathrm{Si}_{2}$. Samples were cleaved in-situ at room temperature in ultrahigh vacuum. Temperature-dependent measurements were performed in the range of $2-22 \mathrm{~K}$ in a cyclic manner.

1. Palstra, T. T. M. et al. Superconducting and Magnetic Transitions in the HeavyFermion System URu $\mathrm{Si}_{2}$. Phys. Rev. Lett. 55, 2727-2730 (1985).

2. Schlabitz, W. et al. Superconductivity and magnetic order in a strongly interacting fermi-system: $\mathrm{URu}_{2} \mathrm{Si}_{2}$. Z. Phys. B Con. Mat. 62, 171-177 (1986).

3. Maple, M. B. et al. Partially gapped Fermi surface in the heavy-electron superconductor $\mathrm{URu}_{2} \mathrm{Si}_{2}$. Phys. Rev. Lett. 56, 185-188 (1986).

4. Broholm, C. et al. Magnetic excitations and ordering in the heavy-electron superconductor $\mathrm{URu}_{2} \mathrm{Si}_{2}$. Phys. Rev. Lett. 58, 1467-1470 (1987).

5. Amitsuka, H. et al. Pressure-temperature phase diagram of the heavy-electron superconductor $\mathrm{URu}_{2} \mathrm{Si}_{2}$. J Magn. Magn. Mater. 310, 214-220 (2007).

6. Haule, K. \& Kotliar, G. Arrested Kondo effect and hidden order in $\mathrm{URu}_{2} \mathrm{Si}_{2}$. Nat. Phys. 5, 796-799 (2009).

7. Harima, H., Miyake, K. \& Flouquet, J. Why the Hidden Order in $\mathrm{URu}_{2} \mathrm{Si}_{2}$ Is Still Hidden-One Simple Answer. J. Phys. Soc. Jpn. 79, 033705 (2010).

8. Cricchio, F., Bultmark, F., Grånäs, O. \& Nordström, L. Itinerant Magnetic Multipole Moments of Rank Five as the Hidden Order in $\mathrm{URu}_{2} \mathrm{Si}_{2}$. Phys. Rev. Lett. 103, 107202 (2009). 
9. Thalmeier, P. \& Takimoto, T. Signatures of hidden-order symmetry in torque oscillations, elastic constant anomalies, and field-induced moments in $\mathrm{URu}_{2} \mathrm{Si}_{2}$. Phys. Rev. B 83, 165110 (2011).

10. Kusunose, H. \& Harima, H. On the Hidden Order in $\mathrm{URu}_{2} \mathrm{Si}_{2}-$ Antiferro Hexadecapole Order and Its Consequences. J. Phys. Soc. Jpn. 80, 084702 (2011).

11. Elgazzar, S., Rusz, J., Amft, M., Oppeneer, P. M. \& Mydosh, J. A. Hidden order in $\mathrm{URu}_{2} \mathrm{Si}_{2}$ originates from Fermi surface gapping induced by dynamic symmetry breaking. Nat. Mater. 8, 337-341 (2009).

12. Oppeneer, P. et al. Electronic structure theory of the hidden-order material $\mathrm{URu}_{2} \mathrm{Si}_{2}$. Phys. Rev. B 82, 205103 (2010).

13. Dubi, Y. \& Balatsky, A. Hybridization Wave as the "Hidden Order" in $\mathrm{URu}_{2} \mathrm{Si}_{2}$. Phys. Rev. Lett. 106, 086401 (2011).

14. Pépin, C., Norman, M., Burdin, S. \& Ferraz, A. Modulated Spin Liquid: A New Paradigm for $\mathrm{URu}_{2} \mathrm{Si}_{2}$. Phys. Rev. Lett. 106, 106601 (2011).

15. Fujimoto, S. Spin Nematic State as a Candidate of the Hidden Order Phase of $\mathrm{URu}_{2} \mathrm{Si}_{2}$. Phys. Rev. Lett. 106, 196407 (2011).

16. Ikeda, H. et al. Emergent rank-5 nematic order in $\mathrm{URu}_{2} \mathrm{Si}_{2}$. Nat. Phys. 8, 528-533 (2012)

17. Okazaki, R. et al. Rotational Symmetry Breaking in the Hidden-Order Phase of $\mathrm{URu}_{2} \mathrm{Si}_{2}$. Science 331, 439-442 (2011).

18. Schmidt, A. R. et al. Imaging the Fano lattice to 'hidden order' transition in $\mathrm{URu}_{2} \mathrm{Si}_{2}$. Nature 465, 570-576 (2010).

19. Aynajian, P. et al. Visualizing the formation of the Kondo lattice and the hidden order in $\mathrm{URu}_{2} \mathrm{Si}_{2}$. P. Natl. Acad. Sci. 107, 10383-10388 (2010).

20. Villaume, A. et al. Signature of hidden order in heavy fermion superconductor $\mathrm{URu}_{2} \mathrm{Si}_{2}$ : Resonance at the wave vector $Q_{0}=(1,0,0)$. Phys. Rev. B 78, 012504 (2008).

21. Hassinger, E. et al. Similarity of the Fermi Surface in the Hidden Order State and in the Antiferromagnetic State of $\mathrm{URu}_{2} \mathrm{Si}_{2}$. Phys. Rev. Lett. 105, 216409 (2010).

22. Santander-Syro, A. F. et al. Fermi-surface instability at the 'hidden-order' transition of $\mathrm{URu}_{2} \mathrm{Si}_{2}$. Nat. Phys. 5, 637-641 (2009).

23. Yoshida, R. et al. Signature of hidden order and evidence for periodicity modification in $\mathrm{URu}_{2} \mathrm{Si}_{2}$. Phys. Rev. B 82, 205108 (2010).

24. Chatterjee, S. et al. Formation of the Coherent Heavy Fermion Liquid at the Hidden Order Transition in $\mathrm{URu}_{2} \mathrm{Si}_{2}$. Phys. Rev. Lett. 110, 186401 (2013).

25. Boariu, F. L. et al. Momentum-Resolved Evolution of the Kondo Lattice into "Hidden Order" in $\mathrm{URu}_{2} \mathrm{Si}_{2}$. Phys. Rev. Lett. 110, 156404 (2013).

26. Denlinger, J. D. et al. Comparative study of the electronic structure of $\mathrm{XRu}_{2} \mathrm{Si}_{2}$ probing the Anderson lattice. J. Electron Spectrosc. 117, 347-369 (2001).

27. Boariu, F. L. et al. The surface state of $\mathrm{URu}_{2} \mathrm{Si}_{2}$. J. Electron Spectrosc. 181, 82-87 (2010).

28. Schoenes, J., Schönenberger, C., Franse, J. \& Menovsky, A. A. Hall-effect and resistivity study of the heavy-fermion system $\mathrm{URu}_{2} \mathrm{Si}_{2}$. Phys. Rev. B 35, 5375-5378 (1987).

29. Bonn, D. A., Garrett, J. D. \& Timusk, T. Far-Infrared Properties of $\mathrm{URu}_{2} \mathrm{Si}_{2}$. Phys. Rev. Lett. 61, 1305-1308 (1988)
30. Hashimoto, M. et al. Particle-hole symmetry breaking in the pseudogap state of Bi2201. Nat. Phys. 6, 414-418 (2010).

31. Van Dijk, N. H. et al. Specific heat of heavy-fermion $\mathrm{URu}_{2} \mathrm{Si}_{2}$ in high magnetic fields. Phys. Rev. B 56, 14493 (1997).

32. Dakovski, G. L. et al. Anomalous femtosecond quasiparticle dynamics of hidden order state in $\mathrm{URu}_{2} \mathrm{Si}_{2}$. Phys. Rev. B 84, 161103 (2011)

33. Hoshino, S., Otsuki, J. \& Kuramoto, Y. Resolution of Entropy $\ln 2$ by Ordering in Two-Channel Kondo Lattice. J. Phys. Soc. Jpn. 82, 044707 (2013).

34. Aoki, D. et al. Field Reentrance of the Hidden Order State of $\mathrm{URu}_{2} \mathrm{Si}_{2}$ under Pressure. J. Phys. Soc. Jpn. 78, 053701 (2009).

35. Aoki, D. et al. Field re-entrant hidden-order phase under pressure in $\mathrm{URu}_{2} \mathrm{Si}_{2}$. J. Phys.: Condens. Matter 22, 164205 (2010).

\section{Acknowledgements}

We thank T. Kim, K. Ott, A. Varykhalov, and E. Rienks for assistance with the measurements. We also thank Y. Haga, Y. Onuki, K. Machida, and S. Hoshino for the valuable discussions. The ARPES measurements were performed at the 'One-Cubed' ARPES station at BESSY II under proposal numbers 2011_2_110195 and 2012_2_120346. This work was supported by a Grant-in-Aid for Scientific Research on Innovative Areas 'Heavy Electrons' (20102003) from the Ministry of Education, Culture, Sports, Science and Technology, a Grant-in-Aid for Scientific Research from the Japan Society for the Promotion of Science (JSPS), and ERC starting grant (NewHeavyFermion). R.Y. also acknowledges the financial support from the Research Fellowships of JSPS for Young Scientists.

\section{Author contributions}

The ARPES data were acquired and analyzed by R.Y. and T.Y. with support from K.T., T.I., M.S., J.S. and T.W. The high-quality single crystals were grown by D.A. and J.F. R.Y. and T.Y. designed the experiment and wrote the manuscript with the help of Y.M.

\section{Additional information}

Supplementary information accompanies this paper at http://www.nature.com/ scientificreports

Competing financial interests: The authors declare no competing financial interests.

How to cite this article: Yoshida, R. et al. Translational Symmetry Breaking and Gapping of Heavy-Quasiparticle Pocket in $\mathrm{URu}_{2} \mathrm{Si}_{2}$. Sci. Rep. 3, 2750; DOI:10.1038/srep02750 (2013).

(c) (i) (2) This work is licensed under a Creative Commons AttributionBY NC SA NonCommercial-ShareAlike 3.0 Unported license. To view a copy of this license, visit http://creativecommons.org/licenses/by-nc-sa/3.0 